\title{
EEG/MEG Spatio-temporal Dipole Source Estimation in Unknown Spatially Correlated Noise ${ }^{\dagger}$
}

\author{
Aleksandar Dogandžić and Arye Nehorai \\ EECS Department (M/C 154), The University of Illinois at Chicago \\ 851 South Morgan Street, Chicago IL 60607, USA \\ email: adogandz@eecs.uic.edu and nehorai@eecs.uic.edu
}

\begin{abstract}
We present maximum likelihood (ML) methods for estimating evoked dipole responses using electroencephalography (EEG) and magnetoencephalography (MEG) arrays, which allow for spatially correlated noise between sensors with unknown covariance. The electric source is modeled as a collection of current dipoles at fixed locations and the head as a spherical conductor. We permit correlation between the dipoles' moments. The dipoles' locations and moments are estimated. We also propose ML-based methods for scanning the brain response data, which can be used for imaging the brain's electromagnetic activity.
\end{abstract}

\section{Introduction}

The non-invasive techniques of electroencephalography (EEG) and magnetoecephalography (MEG) are necessary for understanding both the spatial and temporal behavior of the brain. Arrays of EEG and MEG sensors measure electric potential on the scalp and magnetic field around the head, respectively. These two fields are generated by neuronal activity in the brain, and provide information about both its spatial distribution and temporal dynamics. This is in contrast to other brain imaging techniques that measure anatomical information (MRI, CT), blood flow or blood volume (fMRI, SPECT), or metabolism of oxygen or sugar (PET). Furthermore, the temporal resolution of EEG/MEG is far superior to that achieved by other modalities.

Spatio-temporal EEG/MEG data analysis is based on modeling a source of brain activity by a primary current distributed over a certain region of the cortex. Evoked responses are used to study sensory and cognitive processing

\footnotetext{
†This work was supported by the National Science Foundation under Grant MIP-9615590, the Air Force Office of Scientific Research under Grants F49620-97-1-0481 and F49620-99-1-0067, and the Office of Naval Research under Grant N00014-98-1-0542.
}

in the brain [1], and are applied to clinical diagnosis in neurology and psychiatry. A current dipole is often used as an equivalent source for a uni-directional primary current that may extend over a few square centimeters of cortex. It is justified when the source dimensions are relatively small compared with the distances from the source to the measurement sensors [2], as is often satisfied for sources evoked in response to a given sensory stimulus, e.g. auditory, visual etc.

In [3], spatio-temporal measurements are incorporated using the common dipoles-in-a-sphere model. The dipoles are assumed to have fixed locations and orientations, while their strengths are allowed to change in time according to a parametric model. De Munck [4] extends the above model by allowing the dipole strengths to change arbitrarily. In [5] only the dipole position is fixed and the orientation and amplitude are allowed to vary in time according to a parametric model.

In all the above models, the noise is assumed to be spatially uncorrelated. As a result, these (and most other) localization procedures are based on minimizing a sum of squared errors. Such a residual function is appropriate only if the brain background noise, which is a major source of noise in EEG/MEG, shows no correlation across the scalp at different electrodes. However, since the background noise arises mostly in the cortex, it is expected to be strongly correlated in space. For example, regular rhythms in spontaneous brain activity, such as alpha waves, are not only large in amplitude but also correlated between neighboring sensors [6]. The spatially correlated noise problem is important in EEG because of the bipolar nature of the potential field recordings, i.e. the noise at the reference electrode spreads to all other channels [7]. In MEG, environmental noise is an additional important source of spatial correlation [8], especially in an unshielded environment.

One of the first attempts to tackle the problem of correlated noise was made by Sekihara et al. [8], who assumed known spatial noise covariance. The localization in [8] is 
performed using a generalized least squares (GLS) method (see also [9, Section III-B] and [10, pp. 448-449]) and measurements at only one point in time. In [11], general detection algorithms are derived for known spatial noise covariance and multiple time snapshots whereas the temporal evolutions of the dipole moments are allowed to vary arbitrarily. Lütkenhöner has analyzed the GLS method for multiple time snapshots and applied it to both simulated and real data in [12] and [13]. The algorithm in [4] is extended in [14] and [15] to account for stationary noise correlated in both space and time. However, the noise covariance of such a process has an extremely large number of parameters that need to be determined. It is often estimated from baseline measurements, i.e. data containing only noise collected before the stimulus is applied, assuming that statistically it does not differ between the baseline and a particular time point of interest. However, this approach is suboptimal, since it does not use the data containing the response for estimating the noise covariance. Furthermore, there are indications that utilizing baseline data may not be justified, since it is hypothesized that the noise covariance changes due to dependence on the state of the subject or visual stimulation [7], [16], [17]. Thus, the noise covariance may need to be estimated only from the data containing the response. Here, we present algorithms that solve this problem efficiently.

An iteratively reweighted generalized least squares (IRGLS) procedure [10, pp. 298-300] is proposed in [7] to estimate the noise covariance matrix and fit the dipole locations at a single time point utilizing multiple trials. It is a two-stage procedure yielding estimates which, if the noise is Gaussian, converge to the maximum likelihood (ML) estimates. This method, however, does not include temporal evolution.

In this paper (see also [9]), we propose spatio-temporal location algorithms for dipoles in spatially correlated noise with unknown covariance. We allow for correlated dipoles by specifying the rank of the dipole moment matrix, which is a measure of the level of correlation between the moment components. In Section 3, we present the concentrated likelihood function which should be maximized to obtain the ML estimates of the dipole locations, requiring only a onestage iterative procedure. Finally, in Section 4, we derive ML-based methods for scanning the brain response data, which can be used directly for imaging the brain's electromagnetic activity, or to initialize the multi-dimensional search required for obtaining the dipole location estimates.

\section{Source and Measurement Models}

\subsection{Source Model}

We model the head as a spherically symmetric conductor locally fitted to the head curvature. Let $p$ be the position of

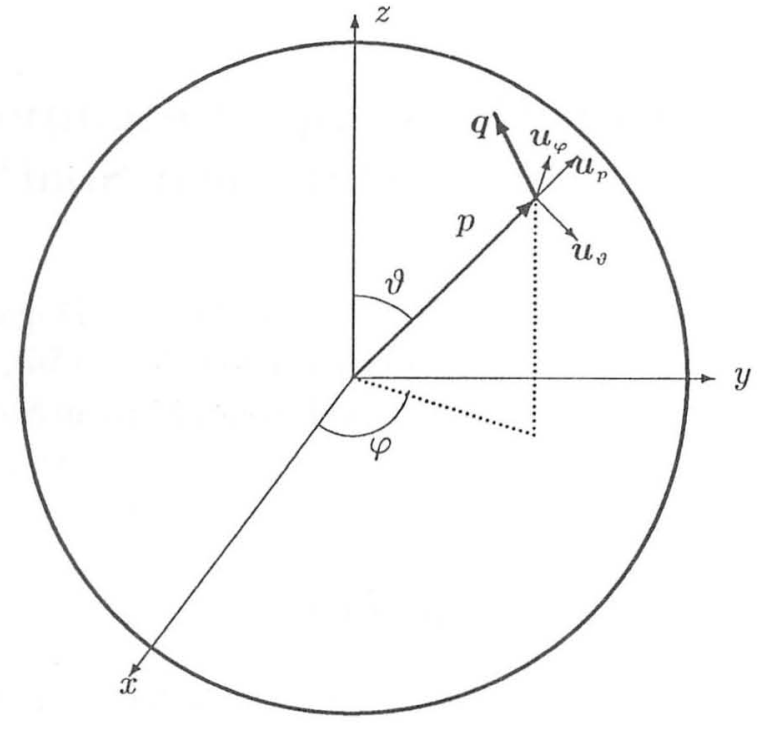

Figure 1. Dipole in a sphere.

a current dipole source relative to the center of the sphere,

$$
p=p[\sin \vartheta \cos \varphi, \sin \vartheta \sin \varphi, \cos \vartheta]^{T},
$$

where $\vartheta$ is the dipole's elevation, $\varphi$ its azimuth, and $p$ its distance from the center, see Figure 1. Thus, $p$ is fully described by $\theta=[\vartheta, \varphi, p]^{T}$. The vectors

$$
\begin{aligned}
& u_{\vartheta}=[\cos \vartheta \cos \varphi, \cos \vartheta \sin \varphi,-\sin \vartheta]^{T}, \\
& u_{\varphi}=[-\sin \varphi, \cos \varphi, 0]^{T}, \\
& u_{p}=p / p
\end{aligned}
$$

form an orthonormal basis (see also [18]). Using this basis, the dipole moment can be written as $q_{\vartheta} u_{\vartheta}+q_{\varphi} u_{\varphi}+$ $q_{p} u_{p}$. We define the vector of moment parameters $q=$ $\left[q_{\vartheta}, q_{\varphi}, q_{p}\right]^{T}$.

\subsection{Measurement Model}

Consider a bimodal array of $m_{\mathrm{E}}$ EEG and $m_{\mathrm{B}}$ MEG sensors. The subscripts E and B refer to the EEG and MEG sensors, respectively. Let $m=m_{\mathrm{E}}+m_{\mathrm{B}}$. Then, the $m$-dimensional measurement vector of this array is

$$
y=A(\theta) q+e,
$$

where $y=\left[y_{\mathrm{B}}^{T}, y_{\mathrm{E}}^{T}\right]^{T}, A(\boldsymbol{\theta})$ is the $m \times 3$ array response matrix and $e=\left[e_{\mathrm{B}}^{T}, e_{\mathrm{E}}^{T}\right]^{T}$ is additive noise. The array response matrix is derived using the quasistatic approximation of Maxwell's equations and spherical head model (see [2] and [19] and references therein). The radial component of a dipole produces no external magnetic field in the spherical head model [2], so the last column of the MEG response matrix is zero. Thus $A(\theta)=$ 
$\left[\left[A_{\mathrm{B}}(\boldsymbol{\theta}), 0_{m_{\mathrm{B}} \times 1}\right]^{T}, A_{\mathrm{E}}(\boldsymbol{\theta})^{T}\right]^{T}$, where $A_{\mathrm{B}}(\theta)$ and $A_{\mathrm{E}}(\theta)$ are the MEG and EEG response matrices with dimensions $m_{\mathrm{B}} \times 2$ and $m_{\mathrm{E}} \times 3$, respectively. The symbol $0_{m_{\mathrm{B}} \times 1}$ denotes the $m_{\mathrm{B}} \times 1$ vector with zero entries. Define $r=$ $\operatorname{rank}(A(\theta))$; usually $r=3$, except when only MEG sensors are employed $(r=2)$.

For $n$ distinct dipoles equation (3) holds with $A(\theta)$, $\theta$, and $q$ substituted with $\left[A\left(\theta_{1}\right) \cdots A\left(\theta_{n}\right)\right], \quad \theta=$ $\left[\boldsymbol{\theta}_{1}^{T} \cdots \boldsymbol{\theta}_{n}^{T}\right]^{T}$, and $\boldsymbol{q}=\left[\boldsymbol{q}_{1}^{T} \cdots \boldsymbol{q}_{n}^{T}\right]^{T}$, respectively. Note that, in this case, $\theta$ and $q$ are $3 n$ - and $r n$-dimensional vectors, respectively. Since the dipoles are at distinct locations, we assume that $A(\theta)$ has full rank equal to $r n$.

The noise vector $\boldsymbol{e}$ is assumed to be zero-mean Gaussian with unknown spatial covariance $\Sigma$, while the source moment signal is deterministic. Thus, the mean and covariance matrix of the snapshot $y$ are $A(\theta) q$ and $\Sigma$, respectively. The noise is predominantly due to background activity in neurons. An assumption of Gaussianity, often used in EEG/MEG literature, may be justified by the additive nature of the noise and the large number of neurons normally active throughout the brain and has been validated in [20]. Tests for the normality of background EEG signals have also been developed in [21].

\section{Simultaneous Estimation of the Dipole Parameters and Noise Covariance}

Assume that the evoked field is a result of brain electrical activity that is well modeled by $n$ dipoles at unknown fixed locations with time-varying moments. As is commonly done in analyzing evoked responses, the experiment is repeated $K$ times to improve the signal-to-noise ratio (SNR). The activated dipoles are assumed to have the same locations and temporal patterns in each experiment, i.e. the evoked responses are homogeneous. In the $k$ th trial ( $k=1, \ldots, K)$, the $N$ temporal data vectors (snapshots) $\boldsymbol{y}_{k}(1), \boldsymbol{y}_{k}(2), \ldots, \boldsymbol{y}_{k}(N)$ are collected. We refer to the matrix $Y_{k}=\left[y_{k}(1) \cdots y_{k}(N)\right]$ as the spatio-temporal data matrix for the $k$ th trial. The measurement model is then

$$
\boldsymbol{y}_{k}(t)=A(\theta) \boldsymbol{q}(t)+e_{k}(t),
$$

for $t=1, \ldots, N$ and $k=1, \ldots, K$. Here $\boldsymbol{e}_{k}(t)$ denotes the noise, which is assumed to be zero mean Gaussian with unknown spatial covariance $\Sigma$, uncorrelated in time and between trials. In reality, the noise is likely to be correlated in time (within a trial), but uncorrelated between trials. The noise covariance matrix $\Sigma$ is assumed to be positive definite and constant in time and across all trials.

Define the spatio-temporal dipole moment matrix as $Q=[q(1) \cdots q(N)]$. To allow for correlated dipole moments, we assume that $Q$ is of rank $l$, where $1 \leq l \leq n r$ [provided that $\operatorname{rank}(A(\theta)) \leq N$, which holds in most practical applications]. If $l=1$, all the components of the dipole moments have the same temporal evolution (up to a scaling factor), thus they are fully correlated. On the other hand, $l=n r$ corresponds to fully uncorrelated dipole moments. For multiple dipoles having fixed orientation (as in e.g. [3] and [4]), each dipole has fully correlated moment components and therefore, provided that the dipole sources are uncorrelated between each other, we may choose $l=n$.

Note that the above model for the dipole moments is equivalent to modeling the spatio-temporal dipole moment matrix as $Q=X B$, where $X$ and $B$ are unknown full-rank matrices with dimensions $n r \times l$ and $l \times N$, respectively. To derive the dipole location algorithms for this model, we start with a (statistically more familiar) model with known $B$, as follows. Define the projection matrix on the row space of $B$ as $P_{B}=B^{T}\left[B B^{T}\right]^{-1} B$. If $K=1$ and $\theta$ and $B$ are known, the above model is known as the generalized multivariate analysis of variance (GMANOVA), first addressed in [22] (see also [23], [24, Chapter 6.4], [10, Chapter 5]). In statistics, it is usually applied to fitting growth curves and thus is also called the growth-curve model [22], [23], [24]. In [9], we extended the GMANOVA equations to multiple trials, i.e. we have shown that, for known $\theta$ and $B$, the ML estimates of $X$ and $\Sigma$ are

$$
\begin{aligned}
\widehat{X} & =\left[A(\boldsymbol{\theta})^{T} S^{-1} A(\theta)\right]^{-1} A(\theta)^{T} S^{-1} \bar{Y} B^{T}\left[B B^{T}\right]^{-1}, \\
\widehat{\Sigma}(\boldsymbol{\theta}, B) & =S+\left(I_{m}-T S^{-1}\right) S_{1}\left(I_{m}-T S^{-1}\right)^{T},
\end{aligned}
$$

where

$$
\begin{aligned}
\bar{Y} & =\frac{1}{K} \sum_{k=1}^{K} Y_{k}, \\
S & =\widehat{R}-S_{1}, \\
\widehat{R} & =\frac{1}{N K} \sum_{k=1}^{K} Y_{k} Y_{k}^{T}, \\
S_{1} & =\frac{1}{N} \bar{Y} P_{B} \bar{Y}^{T}, \\
T & =A(\theta)\left[A(\theta)^{T} S^{-1} A(\theta)\right]^{-1} A(\theta)^{T},
\end{aligned}
$$

and $I_{m}$ denotes the identity matrix of size $m$. Note that $S$ and $S_{1}$ are functions of $B$ only, and $T$ and $\widehat{X}$ are functions of both $\theta$ and $B$. To simplify the notation, we omit these dependencies throughout this paper. For the above model the sufficient statistics are $\bar{Y}$ and $\widehat{R}$. If the matrices $Y_{k}$ become scalars, i.e. $Y_{k}=y_{k}$ and $A(\theta)=a$, we obtain the well known results from univariate statistics $\widehat{\Sigma}=S=1 / K \sum_{k=1}^{K}\left(y_{k}-\bar{y}\right)^{2}$ and $\widehat{X}=\bar{y} / a$.

If, in addition to $X$ and $\Sigma, \theta$ is not known (and $B$ is known), its ML estimate $\widehat{\boldsymbol{\theta}}$ is obtained by maximizing the concentrated likelihood function

$$
l(\theta, B)=\frac{\left|B\left(I_{N}-\frac{1}{N} \bar{Y}^{T} W(\theta) \bar{Y}\right) B^{T}\right|}{\left|B\left(I_{N}-\frac{1}{N} \bar{Y}^{T} \widehat{R}^{-1} \bar{Y}\right) B^{T}\right|}
$$




$$
=\frac{\left|A(\theta)^{T} S^{-1} A(\theta)\right|}{\left|A(\theta)^{T} \widehat{R}^{-1} A(\theta)\right|}
$$

where

$$
W(\theta)=\widehat{R}^{-1}-\widehat{R}^{-1} A(\theta)\left[A(\theta)^{T} \widehat{R}^{-1} A(\theta)\right]^{-1} A(\theta)^{T} \widehat{R}^{-1}
$$

The above result is obtained by replacing $X$ and $\Sigma$ in the likelihood function with their ML estimates in (5), equation (11) having been derived in [9, eq. (C.2)] (see also [25, pp. 26-28]), and equation (12) easily following from results of [9, App. A] and [25, p. 24]. Once $\widehat{\theta}$ is obtained, the ML estimates of $X$ and $\Sigma$ follow by replacing $\theta$ in expressions (5) with $\hat{\boldsymbol{\theta}}$.

Consider now the case when both $X$ and $B$ are unknown and only their rank $l$ is specified. [This is equivalent to assuming that the spatio-temporal dipole moment matrix $Q$ is unknown with rank $l$.] Starting from (11) and using the Poincaré separation theorem [26, pp. 64-65], we have shown in [9] that the ML estimate of $\boldsymbol{\theta}$ is obtained by maximizing the concentrated likelihood function in the form of a product of the $l$ largest generalized eigenvalues of the matrices $I_{N}-\frac{1}{N} \bar{Y}^{T} W(\theta) \bar{Y}$ and $I_{N}-\frac{1}{N} \bar{Y}^{T} \widehat{R}^{-1} \bar{Y}$. Also, we have shown that the ML estimates of $X$ and $B$ are not unique; however the estimated dipole moment matrix $\widehat{Q}=\widehat{X} \widehat{B}$ is unique (see [9, eqs. (C.6) and (C.8)] for the exact expression for $\widehat{Q}$ ). If $l=n r$, i.e. the dipole moments are fully uncorrelated, the concentrated likelihood is simply

$$
l(\theta)=\frac{\left|I_{N}-\frac{1}{N} \bar{Y}^{T} W(\theta) \bar{Y}\right|}{\left|I_{N}-\frac{1}{N} \bar{Y}^{T} \widehat{R}^{-1} \bar{Y}\right|},
$$

which follows from the fact that the determinant of a matrix is equal to the product of its eigenvalues. Further, this expression is equal to the concentrated likelihood in (11) when $B=I_{N}$.

\section{Scanning}

Using the ML estimation results in Section 3, we derive two scanning schemes, based on maximizing suitably chosen functions of the data and the single-dipole array response (thus reducing the dimensionality of the problem compared with the multiple-dipole location algorithms). In the EEG/MEG literature, scanning has often been performed using the MUSIC algorithm [27]. However, MUSIC does not perform well when the sources are correlated [28], [29] or the noise is spatially correlated [30], or both. The scanning algorithms proposed here take into account spatially correlated noise with unknown covariance. The first scheme consists of maximizing the concentrated likelihood function for a single dipole. The second scheme is based on matching the estimated array response subspace with a single-dipole array response.
If the dipole moments are assumed to be uncorrelated, the first scanning scheme reduces simply to computing (14) using the array response $A(\theta)$ for a single dipole. For a source with fixed orientation, the dipole moments are fully correlated, and the rank of the moment matrix is $l=1$. Then, the concentrated likelihood is $l(\theta)=\lambda_{\operatorname{MAx}}\left(I_{N}-\right.$ $\left.\frac{1}{N} \bar{Y}^{T} W(\theta) \bar{Y}, I_{N}-\frac{1}{N} \bar{Y}^{T} \widehat{R}^{-1} \bar{Y}\right)$, where $\lambda_{\text {MAx }}(\cdot, \cdot)$ denotes the largest generalized eigenvalue of the two matrices given in the parenthesis. The above scanning procedures require only a 3-D search over the dipole location parameters. They are intuitively appealing, since they evaluate the likelihood of a dipole at a particular location, while simultaneously estimating the unknown noise covariance, which accounts for the sources of brain activity at other locations.

If a realistic head model is used, we can impose anatomic constraints [31], i.e. assume that sources can lie only on the surface of the cortex with moments orthogonal to the cortex. Then, we can recast the array response matrix by incorporating the known orientation, which gives a response vector $\boldsymbol{a}(\boldsymbol{\theta})$, where $\theta$ is a $2 \times 1$ parameter vector describing a location on the surface of the cortex. In this case, the dimensionality of the search is only 2-D, and the scanning function simplifies to

$$
\begin{aligned}
l(\theta)= & 1+\frac{1}{N a(\theta)^{T} \widehat{R}^{-1} a(\theta)} \cdot a(\theta)^{T} \widehat{R}^{-1} \bar{Y} \\
& \times\left[I_{N}-\frac{1}{N} \bar{Y}^{T} \widehat{R}^{-1} \bar{Y}\right]^{-1} \bar{Y}^{T} \widehat{R}^{-1} a(\theta)
\end{aligned}
$$

after substituting $A(\theta)=a(\theta)$ in (14) and using the formula for the determinant of a partitioned matrix (see e.g. $[10$, result v, p. 8]). The performance of the above scanning method relies on the validity of the above constraints and would require using patient-specific MRI images to extract the necessary information (e.g. the surface of the cortex). Observe that the expression (15) has a Capon-like structure [32], [33] in the denominator.

We now derive the second scanning scheme based on matching the estimated array response subspace (constructed using an ML estimate of non-parametric array response) with a single-dipole array response. The matching is performed using a MUSIC-like function .

Consider the non-parametric array response model, i.e. assume that $A(\theta)=A$ is an unknown $m \times n r$ matrix of full rank, equal to $n r$ (since, in practical applications, $m>n r$ ) see also [25, p. 25], [34]. Also, assume that the dipole moments are fully uncorrelated, i.e. $B=I_{N}$. Then, equation (7) yields $S=\widehat{R}-\frac{1}{N} \overline{Y Y}^{T}$. In the following, we compute an ML estimate of $A$ by maximizing the concentrated likelihood function in (12) [where $A(\theta)$ is replaced with $A$ ] with respect to $A$.

Denote by $\widehat{R}^{\frac{1}{2}}$ a symmetric square root of a symmetric matrix $\widehat{R}$, and let $\widehat{R}^{-\frac{1}{2}}=\left(\widehat{R}^{\frac{1}{2}}\right)^{-1}$; this notation will 
be used throughout. Consider the array response matrix of the form $A=\widehat{R}^{\frac{1}{2}} V C$, where $C$ is an $m \times n r$ matrix of full rank $n r$ and $V$ is the matrix whose columns are the (normalized) eigenvectors of $\Xi=\frac{1}{N} \widehat{R}^{-\frac{1}{2}} \overline{Y Y}^{T} \widehat{R}^{-\frac{1}{2}}$ that are ordered to correspond to the eigenvalues of $\Xi$ (denoted by $\lambda_{j}, j=1, \ldots, m$ ) sorted in non-increasing order, i.e. $\lambda_{1} \geq \lambda_{2} \cdots \geq \lambda_{m}$. Thus, $\Xi=V \operatorname{diag}\left\{\lambda_{1}, \ldots, \lambda_{m}\right\} V^{T}$. Then, the concentrated likelihood function in (12) becomes

$$
\begin{aligned}
l(\theta) & =\frac{\left|C^{T} V^{T} \widehat{R}^{\frac{1}{2}}\left(\widehat{R}-\frac{1}{N} \overline{Y Y}^{T}\right)^{-1} \widehat{R}^{\frac{1}{2}} V C\right|}{\left|C^{T} C\right|} \\
& =\frac{\left|C^{T}\left[I_{m}-\operatorname{diag}\left\{\lambda_{1}, \ldots, \lambda_{m}\right\}\right]^{-1} C\right|}{\left|C^{T} C\right|},
\end{aligned}
$$

which follows from

$\widehat{R}^{\frac{1}{2}}\left(\widehat{R}-\frac{1}{N} \overline{Y Y}^{T}\right)^{-1} \widehat{R}^{\frac{1}{2}}=V\left[I_{m}-\operatorname{diag}\left\{\lambda_{1}, \ldots, \lambda_{m}\right\}\right]^{-1} V^{T}$

Since $0 \leq\left(\bar{Y}^{T} \widehat{R}^{-1} \bar{Y}\right) / N \leq I_{N}$, the non-zero eigenvalues of $\left(\bar{Y}^{T} \widehat{R}^{-1} \bar{Y}\right) / N$ (equal to the non-zero eigenvalues of $\Xi$ ) are between 0 and 1 ; thus, $0 \leq \lambda_{j} \leq 1$, where $j=1, \ldots, m$. Note that $1 /\left(1-\lambda_{1}\right) \geq 1 /\left(1-\lambda_{2}\right) \cdots \geq$ $1 /\left(1-\lambda_{m}\right)$, because $1 /\left(1-\lambda_{j}\right)$ is an increasing function of $\lambda_{j}$ for $0 \leq \lambda_{j}<1$, Now, it is obvious that (16) is maximized for $C=\left[I_{n r}, 0\right]^{T} \cdot H$ where $H$ is an arbitrary $n r \times n r$ matrix of full rank. Thus, an ML estimate of $A$ is of the following form: $\widehat{A}=\widehat{R}^{\frac{1}{2}} V_{n r} H$, where $V_{n r}$ denotes the matrix containing the first $n r$ columns of $V$. Further, $I_{m}-V_{n r} V_{n r}{ }^{T}$ is the projection matrix onto the space orthogonal to the column space of $\widehat{R}^{-\frac{1}{2}} \widehat{A}$. Therefore, for a single dipole located at $\boldsymbol{\theta}$,

$$
\left(I-V_{n r} V_{n r}^{T}\right) \widehat{R}^{-\frac{1}{2}} A(\theta) \approx 0,
$$

and a MUSIC-like scanning function easily follows (using e.g. [27]) as an inverse of the minimum generalized eigenvalue of $A(\theta)^{T} \widehat{R}^{-\frac{1}{2}}\left[I-V_{n r} V_{n r}{ }^{T}\right] \widehat{R}^{-\frac{1}{2}} A(\theta)$ and $A(\theta)^{T} \widehat{R}^{-1} A(\theta)$. Note also that the columns of $U_{n r}=$ $\widehat{R}^{-\frac{1}{2}} V_{n r}$ are the generalized eigenvectors of $\frac{1}{N} \overline{Y Y}^{T r}$ and $\widehat{R}$ corresponding to their $n r$ largest generalized eigenvalues (equal to $\lambda_{1}, \lambda_{2}, \ldots, \lambda_{n r}$ ); thus $A(\theta)^{T} \widehat{R}^{-\frac{1}{2}}\left[I_{m}-\right.$ $\left.V_{n r} V_{n r}{ }^{T}\right] \widehat{R}^{-\frac{1}{2}} A(\theta)=A(\boldsymbol{\theta})^{T}\left[\widehat{R}^{-1}-U_{n r} U_{n r}{ }^{T}\right] A(\boldsymbol{\theta})$. То summarize, a MUSIC-like scanning function is:

$$
l(\theta)=\frac{1}{\lambda_{\mathrm{MIN}}\left(A(\boldsymbol{\theta})^{T}\left[\widehat{R}^{-1}-U_{n r} U_{n r}^{T}\right] A(\theta), A(\theta)^{T} \widehat{R}^{-1} A(\theta)\right)},
$$

where

- $\lambda_{\text {MIN }}(\cdot, \cdot)$ denotes the smallest generalized eigenvalue of the two matrices given in the parenthesis,

- $A(\theta)$ is a single-dipole array response matrix, and
- $U_{n r}$ is the matrix whose columns are the generalized eigenvectors of $\frac{1}{N} \overline{Y Y}^{T}$ and $\widehat{R}$, corresponding to their largest $n r$ generalized eigenvalues (normalized such that $U_{n r}{ }^{T} \widehat{R} U_{n r}=I_{n r}$ ).

Unlike the first scanning scheme, this method requires specifying the number of dipoles $n$.

Our preliminary simulation results (see also [9]) show good performance of the above scanning schemes, particularly in coping with spatially correlated noise.

\section{Concluding Remarks}

We proposed maximum likelihood methods for estimating evoked dipole responses using a combination of EEG and MEG arrays, assuming spatially correlated noise with unknown covariance. We further derived ML-based methods for scanning the brain response data. We are currently working on extending these methods to more realistic noise and signal models (e.g. temporally correlated noise, latency jitters, patient-specific head models obtained from MRI scans [35], etc).

\section{References}

[1] J. Raz and B. Turetsky, "Event-related potentials," in Encyclopedia of Biostatistics, vol. 2, pp. 1407-1409, Chichester, UK: Wiley, 1998.

[2] M.S. Hämäläinen, R. Hari, R. Ilmoniemi, J. Knuutila, and O.V. Lounasmaa, "Magnetoencephalographytheory, instrumentation, and applications to noninvasive studies of signal processing of the human brain," Rev. Mod. Phys., vol. 65, no. 2, pp. 413-497, Apr. 1993.

[3] M. Scherg and D. Von Cramon, "Two bilateral sources of the late AEP as identified by a spatio-temporal dipole model," Electroencephalogr. Clin. Neurophysiol., vol. 62, pp. 32-44, 1985.

[4] J.C. de Munck, "The estimation of time varying dipoles on the basis of evoked potentials," Electroencephalogr: Clin. Neurophysiol., vol. 77, pp. 156-160, 1990.

[5] M. Scherg and D. Von Cramon, "Evoked dipole source potentials of the human auditory cortex," Electroencephalogr. Clin. Neurophysiol., vol. 65, pp. 344-360, 1986.

[6] C. Braun, S. Kaiser, W.E. Kincses, and T. Elbert, "Confidence interval of single dipole locations based on EEG data," Brain Topogr:, vol. 10, no. 1, pp. 31-39, 1997.

[7] H.M. Huizenga, P.C.M. Molenaar, "Equivalent source estimation of scalp potential fields contaminated by heteroscedastic and correlated noise," Brain Topogr., vol. 8, no. 1, pp. 13-33, 1995. 
[8] K. Sekihara, Y. Ogura, and M. Hotta, "Maximumlikelihood estimation of current-dipole parameters for data obtained using multichannel magnetometer," IEEE Trans. Biomed. Eng., vol. 39, no. 6, pp. 558-562, June 1992.

[9] A. Dogandžić and A. Nehorai, "Estimating evoked dipole responses in unknown spatially correlated noise with EEG/MEG arrays," IEEE Trans. Signal Processing, vol. 48, pp. 13-25, Jan. 2000.

[10] E.F. Vonesh and V.M. Chinchilli, Linear and Nonlinear Models for the Analysis of Repeated Measurements, New York: Marcel Dekker, 1997.

[11] A. Dogandžić and A. Nehorai, "Detecting a dipole source by MEG/EEG and generalized likelihood ratio tests," Proc. 30th Asilomar Conf. Signals, Syst. Comput., Pacific Grove, CA, Nov. 1996, pp. 1196-1200.

[12] B. Lütkenhöner, "Dipole source localization by means of maximum likelihood estimation I. Theory and simulations," Electroencephalogr. Clin. Neurophysiol., vol. 106, pp. 314-321, 1998.

[13] — , "Dipole source localization by means of maximum likelihood estimation II. Experimental evaluation," Electroencephalogr. Clin. Neurophysiol., vol. 106, pp. 322-329, 1998.

[14] T. Yamazaki, B.W. van Dijk, and H. Spekreijse, "Confidence limits for the parameter estimation in the dipole localization method on the basis of spatial correlation of background EEG," Brain Topogr., vol. 5, no. 2, pp. 195-198, 1992.

[15] T. Yamazaki, B.W. van Dijk, and H. Spekreijse, "The accuracy of localizing equivalent dipoles and the spatio-temporal correlations of background EEG," IEEE Trans. Biomed. Eng., vol. 45, pp. 1114-1121, Sept. 1998.

[16] J.C. de Munck, P.C. Vijn and F.H. Lopes da Silva, "A random dipole model for spontaneous brain activity," IEEE Trans. Biomed. Eng., vol. 39, no. 8, pp. 791-804, Aug. 1992.

[17] P.C. Vijn, B.W. van Dijk, and H. Spekreijse, "Topography of occipital EEG-reduction upon visual stimulation," Brain Topogr., vol. 5, no. 2, pp. 177-181, 1992.

[18] B. Hochwald and A. Nehorai, "Magnetoencephalography with diversely-oriented and multi-component sensors," IEEE Trans. Bionled. Eng., vol. 44, pp. 40-50, Jan. 1997.

[19] Z. Zhang, "A fast method to compute surface potentials generated by dipoles within multilayer anisotropic spheres," Phys. Med. Biol., vol. 40, pp. 335-349, 1995.

[20] T. Gasser, J. Möchs, and W. Köhler, "Amplitude probability distribution of noise for flash-evoked potentials and robust response estimates," IEEE Trans. Biomed. Eng., vol. BME-33, pp. 579-584, June 1986.
[21] J.A. McEwen, and G.B. Anderson, "Modeling the stationarity and Gaussianity of spontaneous electroencephalographic activity," IEEE Trans. Biomed. Eng., vol. BME-22, pp. 361-369, Sep. 1975.

[22] R.F. Potthoff and S.N. Roy, "A generalized multivariate analysis of variance model useful especially for growth curve problems," Biometrika, vol. 51, pp. 313 326, 1964.

[23] C.G. Khatri, "A note on a MANOVA model applied to problems in growth curve," Ann. Inst. Statist. Math., vol. 18, pp. 75-86, 1966.

[24] M.S. Srivastava and C.G. Khatri, An Introduction to Multivariate Statistics, New York: North Holland, 1979.

[25] E.J. Kelly and K.M. Forsythe, "Adaptive detection and parameter estimation for multidimensional signal models," Lincoln Lab, Mass. Inst. Technol., Cambridge, MA, Tech. Rep. 848, Apr. 1989.

[26] C.R. Rao, Linear Statistical Inference and Its Applications, New York: Wiley, 2nd ed., 1973.

[27] J.C. Mosher, P.S. Lewis, and R.M. Leahy, "Multiple dipole modeling and localization from spatio-temporal MEG data," IEEE Trans. Biomed. Eng., vol. 39, pp. 541-557, June 1992.

[28] P. Stoica and A. Nehorai, "MUSIC, maximum likelihood and Cramér-Rao bound," IEEE Trans. Acoust., Speech, Signal Processing, vol. 37, pp. 720-741, May 1989.

[29] ——, "MUSIC, maximum likelihood and Cramér-Rao bound - further results and comparisons," IEEE Trans. Acoust., Speech, Sign. Processing, vol. 38, pp. 21402150, Dec. 1990.

[30] P. Stoica, M. Viberg, and B. Ottersten, "Instrumental variable approach to array processing in spatially correlated noise fields," IEEE Trans. Signal Processing, vol. 42, pp. 121-133, Jan. 1994.

[31] A.M. Dale and M.I. Sereno, "Improved localization of cortical activity by combining EEG and MEG with MRI cortical surface reconstruction: a linear approach," J. Cognit. Neurosci., vol. 5, no. 2, pp. 162-176, 1993.

[32] D.H. Johnson and D.E. Dudgeon, Array Signal Processing, Englewood Cliffs, NJ: Prentice Hall, 1993.

[33] K. Sekihara and B. Scholz, "Generalized Wiener estimation of three-dimensional current distribution from biomagnetic measurements," IEEE Trans. Biomed. Eng., vol. 43, pp. 281-291, March 1996.

[34] P. Stoica and M. Viberg, "Maximum likelihood parameter and rank estimation in reduced-rank multivariate linear regressions," IEEE Trans. Signal Processing, vol. 44, pp. 3069-3078, Dec. 1996.

[35] C.H. Muravchik and A. Nehorai, "MEG/EEG numerical error bounds for a dipole source with a realistic head model" Proc. 19th Annu. Int. Conf. IEEE Eng. Med. Biol. Soc., Chicago, IL, Oct. 1997, pp. 1233-1236. 